\title{
Optimization of groove texture profile to improve hydrodynamic lubrication performance: Theory and experiments
}

\author{
Wei WANG ${ }^{1,2}$, Yongyong $\mathrm{HE}^{1, *}$, Jun ZHAO ${ }^{1}$, Junyuan MAO ${ }^{1}$, Yutong HU ${ }^{1}$, Jianbin LUO ${ }^{1}$ \\ ${ }^{1}$ State Key Laboratory of Tribology, Tsinghua University, Beijing 100084, China \\ ${ }^{2}$ Intelligent \& Connected Vehicle Testing Department, China Software Testing Center, Beijing 100048, China \\ Received: 07 May 2018 / Revised: 16 July 2018 / Accepted: 17 September 2018 \\ (C) The author(s) 2018. This article is published with open access at Springerlink.com
}

\begin{abstract}
It is well known that groove texture with a careful design can be used to enhance the load-carrying capacity of oil film under the conditions of hydrodynamic lubrication. In this study, a general parametric model was developed, and agenetic algorithm-sequential quadratic programming hybrid method was adopted to obtain the global-optimum profile of the groove texture. The optimized profiles at different rotating speeds are all chevrons. The numerical analysis results verified the effect of the optimization. In addition to the numerical optimization, experiments were conducted to validate the superiority of the optimized results.The experimental results show that the optimized groove texture can efficiently reduce the coefficient of friction (COF) and the temperature rise of the specimen. In particular, the optimized groove textures can achieve stable ultra-low COF values $(\mathrm{COF}<0.01)$ under certain conditions.
\end{abstract}

Keywords: groove texture; hydrodynamic lubrication; laser texturing; ultra-low coefficient of friction

\section{Introduction}

Surface texturing, which generally involves manufacturing grooves and dimples at the micrometer scale, can be applied to improve the hydrodynamic lubrication performance of mechanical components. Surface texture with a careful design may offer significant improvement in load-carrying capacity (LCC) [1-3], reduction in the coefficient of friction (COF) $[4,5]$ and enhanced resistance to wear [6, 7]. For this reason, surface texturing has been attracting increasing attention from researchers as an efficient surface modification approach.

The geometry of the surface texture may have a strong influence on hydrodynamic lubrication performance metrics such as LCC and COF. Thus, geometry optimization design is critical to guarantee the performance of the surface texture. The bulk of experimental and numerical studies have been devoted to improving the tribological characteristics of textured surfaces by optimization. Generally, the optimization design works mainly concentrate on four aspects: shape, orientation, size, and distribution [8].

The trial-and-error approach is a common method for the optimization of a surface texture's shape [9]. Siripuram et al. [10] investigated different shapes of surface textures for sliding surface lubrication, such as circle, square, diamond, hexagon, and triangle. They concluded that the friction coefficient is not obviously influenced by the shape and orientation, but it is highly sensitive to the area ratio of the surface texture. $\mathrm{Yu}$ et al. [11] used the successive over relaxation method to obtain the average hydrodynamic pressure generated by the dimples in shapes of circles, ellipses, and triangles. They concluded that the LCC is obviously influenced by the geometric shape and the orientation of the surface texture. Ren [12] developed a numerical model for mixed lubrication, and five groups of texture shapes, i.e., fishbone with grooves, fishbone with dimples, sinusoidal waves,

* Corresponding author: Yongyong HE, E-mail: heyy@mail.tsinghua.edu.cn 


\begin{tabular}{|llll}
\multicolumn{2}{l}{ Nomenclature } & & \\
$\mathrm{AR}$ & Area ratio & $p_{\mathrm{c}}$ & Cavitation pressure \\
$d_{\mathrm{g}}$ & Depth of groove texture & $r_{,}, \theta, z$ & Column coordinates \\
$D_{\mathrm{o}}$ & Outside diameter of bottom ring & $r_{\mathrm{i}}$ & Inside radius of the ring \\
$D_{\mathrm{i}}$ & Inside diameter of bottom ring & $r_{\mathrm{o}}$ & Outside radius of the ring \\
$d_{\mathrm{o}}$ & Outside diameter of upper ring & $T$ & Surface temperature \\
$d_{\mathrm{i}}$ & Inside diameter of upper ring & $\Lambda$ & Groove depth ratio \\
$F_{\mathrm{f}}$ & Friction force & $H$ & Fluid viscosity \\
$H$ & Oil film thickness & $\theta_{0}$ & Unit cell angle \\
$h_{0}$ & Minimum oil film thickness & $\theta_{1}$ & Groove width angle \\
$n_{\mathrm{g}}$ & Node number of the grid & $\Theta_{1}, \Theta_{2,} \ldots, \Theta_{\mathrm{n}+1}$ & Design variables \\
$n_{\mathrm{t}}$ & Number of groove texture & $T$ & Shear stress \\
$N$ & Local optimum interval & $\psi$ & Film content \\
$P$ & Pressure & $\Omega$ & Rotating speed \\
$p_{0}$ & Ambient pressure & &
\end{tabular}

triangular grooves, and honeycombs, were investigated numerically. Their results indicated that short grooves with a small aspect ratio and sinusoidal waves with a small wavelength/amplitude ratio obtain the strongest LCC. Yao et al. [13] investigated the aerostatic and aerodynamic performance of herringbone groove textures for gas-lubricated thrust bearings. They found that the herringbone grooves are preferred over spiral grooves considering the bearing stiffness, airflow, and orifice pressure ratio.

In addition to the trial-and-error method, general parametric models provide a new research path for the optimization of surface textures. Fesanghary et al. [14] established the parametric model of the groove texture and optimized the shape using a mathematical method. Their work indicated that the optimum grooves have an elongated "heart-like" shape for small aspect ratios. The experimental results show good agreement with theoretical simulations. Shen et al. $[15,16]$ obtained the optimum texture shape with the sequential quadratic programming (SQP) method, and they observed that the optimum textures have chevron-type shapes with flat fronts for unidirectional sliding. Moreover, their experimental study validated their optimized results. By summarizing these studies, it can be seen that the parametric optimization method is no longer based on pre-determined geometric shapes.

The object of this study was to explore the globaloptimum profile of the groove texture using the parametric optimization method to improve the hydrodynamic lubrication characteristics of the groove texture. A GA-SQP hybrid method was used to solve this multi-parameter nonlinear optimization problem. Compared with the works of Fesanghary et al. and Shen et al., the parametric model was simplified reasonably and efficiently for the optimization. The optimization results can be widely applied to such items as thrust bearings, mechanical seals, and port plate pairs in axial piston pumps.

Section 2 describes how the groove texture was numerically optimized on the basis a general parametric model. Three different profiles were studied experimentally and numerically in order to validate the optimized results. The experimental details, results, and analysis are presented in Sections 3 and 4. Finally, the conclusions are provided in the last section.

\section{Numerical optimization of groove texture}

\subsection{Geometric model}

Figure 1(a) shows a schematic representation of a parallel thrust bearing. The untextured upper surface rotates with a uniform speed. The lower surface with groove textures remains stationary. The column coordinate system is shown in this figure. $\theta$ is the angular coordinate in the rotating direction, $r$ is the coordinate along the radial direction, and $z$ is the coordinate 



(b)

(d)
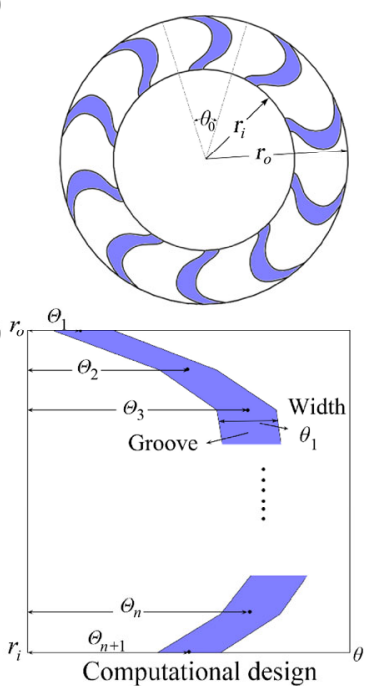

Fig. 1 Numerical model for groove texture: (a) schematic view of a thrust bearing; (b) schematic view of the textured surface; (c) a computing unit cell; and (d) design variables and computational domain for an arbitrary groove shape.

normal to the parallel bearing. The friction pair is fully lubricated by a Newtonian, incompressible lubricant with a constant viscosity. The lubricant fluid is in a laminar flow because the Reynolds number is quite small. These assumptions are normal methods similar to those used in Refs. [11, 17]. The two parallel surfaces are separated by a gap $h_{0}$. The textured surface is shown in Fig. 1(b). On the basis of the periodic boundary condition, a groove is extracted as a computing unit cell for theoretical study, as shown in Fig. 1(c). The boundary conditions of the inlet and outlet of this unit cell are periodic, and the boundary condition of the other sides is the ambient pressure $p_{0}$. The design variables and the computational domain for an arbitrary groove shape are shown in Fig. 1(d). The groove texture is divided into several parts by a series of horizontal lines evenly distributed in the $r$ direction. In this general parametric model, the design variables, $\Theta_{1}, \Theta_{2}, \ldots, \Theta_{n+1}$, are the center locations of the groove texture, which define the flat geometry of the groove texture. The width angle and depth of the groove texture stay constant. In this way, the optimization of the profile of the groove texture is converted to a multi-parameter nonlinear optimization problem. The area ratio $\mathrm{AR}$ is defined as the ratio of the groove width angle $\left(\theta_{1}\right)$ and the unit cell angle $\left(\theta_{0}\right)$, which is

presented in Eq. (1).

$$
A R=\theta_{1} / \theta_{0}
$$

The groove depth ratio $\lambda$ is defined as the ratio of the groove depth $\left(d_{\mathrm{g}}\right)$ and the minimum lubricant film thickness $\left(h_{0}\right)$, which is presented in Eq. (2).

$$
\lambda=d_{\mathrm{g}} / h_{0}
$$

\subsection{Governing equations and cavitation boundary conditions}

Cavitation was considered in this study. At present, there are several common cavitation boundary conditions, i.e., Sommerfeld, half-Sommerfeld, Reynolds, and JFO. Zhang et al. [18] observed the cavitation phenomenon directly, and their experimental results showed that the observed cavitation morphology in steady state is similar to the prediction from the JFO model. In this study, the mass-conserving model proposed by Elord and Adams [19] was adopted to simulate this phenomenon. Under the assumption of a Newtonian and incompressible lubricant, the Reynolds equation in the column coordinate is employed to analyze the pressure distribution.

$$
\frac{1}{r} \frac{\partial}{\partial r}\left(r h^{3} \frac{\partial p}{\partial r}\right)+\frac{1}{r^{2}} \frac{\partial}{\partial \theta}\left(h^{3} \frac{\partial p}{\partial \theta}\right)=6 \eta \omega \frac{\partial \psi h}{\partial \theta}
$$

In Eq. (3), $h$ is the lubricant film thickness, $p$ is the pressure, $\eta$ is the fluid viscosity, $\psi$ is the film content, and $\omega$ is the rotating speed of the upper surface. The dimensionless term of Eq. (3) can be expressed as follows:

$$
\begin{gathered}
\frac{\partial}{\partial \bar{r}}\left(\bar{h}^{3} \frac{\partial \bar{p}}{\partial \bar{r}}\right)+\left(\frac{\theta_{0}}{r_{o}-r_{i}}\right)^{2} \frac{\partial}{\partial \bar{\theta}}\left(\bar{h}^{3} \frac{\partial \bar{p}}{\partial \bar{\theta}}\right)=\Lambda \frac{\partial \psi \bar{h}}{\partial \bar{\theta}}, \\
\bar{r}=\frac{r}{r_{o}-r_{i}}, \bar{\theta}=\frac{\theta}{\theta_{0}}, \bar{h}=\frac{h}{h_{0}}, \bar{p}=\frac{p}{p_{0}}, \Lambda=\frac{6 \eta \omega}{p_{0}}\left(\frac{r_{o}-r_{i}}{h_{0}}\right)^{2}
\end{gathered}
$$

The boundary conditions can be expressed as

$$
\begin{gathered}
\left.\bar{P}\right|_{\bar{r}=\bar{r}_{i}}=1 ;\left.\bar{p}\right|_{\bar{r}=\bar{r}_{o}}=1 ; \\
\left.\bar{P}\right|_{\bar{\theta}=0}=\left.\bar{p}\right|_{\bar{\theta}=1} ;\left.\frac{\partial \bar{p}}{\partial \bar{\theta}}\right|_{\bar{\theta}=0}=\left.\frac{\partial \bar{p}}{\partial \bar{\theta}}\right|_{\bar{\theta}=1}
\end{gathered}
$$


The finite difference method (FDM) was used to discretize Eq. (4), and then it was solved using successive over relaxation. For the detailed solution procedure, refer to the research work by Fesanghary et al. [20].

The uniform basic parameters, such as the side length of the unit cells and the thickness of the oil film, were used for the optimizations in this study. It is noteworthy that this research focused on the influence of the profile of the groove texture rather than the area ratio on the lubrication characteristics, so the optimizations and following experiments in this study adopted a uniform area ratio of 0.25 . These basic parameters are listed in Table 1.

\subsection{Multi-parameter nonlinear optimization algorithm}

The SQP method is an effective algorithm for solving nonlinear optimization problems of small and medium size, and was derived from Wilson's Newton-Lagrange method [21, 22]. However, the optimized results of SQP are sensitive to the initial values. As a result, it is a local optimization algorithm. The genetic algorithm (GA) was first pioneered by John Holland in the 1960s, and since then it has been widely used in various fields. It is an adaptive heuristic algorithm introduced in the evolutionary themes of natural selection and genetic mechanism. In addition, it is a global optimization algorithm. However, the local search efficiency of GA is lower, and the accuracy of the final solution is poor. According to the conclusion of the previous research [8], GA and SQP can be combined to overcome the difficulties that appear when they are used alone.

Table 1 Basic parameters for the optimization.

\begin{tabular}{cc}
\hline Parameter & Value \\
\hline Unit cell angle, $\theta_{0}\left(^{\circ}\right)$ & 11.25 \\
Area ratio, AR & 0.25 \\
Groove depth ratio, $\lambda$ & 1.0 \\
Inside radius of the ring, $r_{\mathrm{i}}(\mathrm{mm})$ & 20.75 \\
Outside radius of the ring, $r_{\mathrm{o}}(\mathrm{mm})$ & 25.25 \\
Node number of the grid, $n_{\mathrm{g}}$ & $64 * 64$ \\
Number of optimization variables, $n+1$ & 9 \\
Minimum film thickness, $h_{0}(\mu \mathrm{m})$ & 5 \\
Ambient pressure, $p_{0}(\mathrm{~Pa})$ & $1.0 \mathrm{e} 5$ \\
Cavitation pressure, $p_{\mathrm{c}}(\mathrm{Pa})$ & $0.3 \mathrm{e} 5$ \\
Viscosity of the oil, $\eta(\mathrm{Pa} \cdot \mathrm{s})$ & 0.038 \\
\hline
\end{tabular}

In this study, the optimization target was the maximization of the LCC of the oil film. The dimensionless form of the LCC is defined as the fitness for the GA and can be obtained by

$$
\overline{L C C}=\frac{\int_{0}^{1} \int_{\bar{r}_{i}}^{\overline{r_{i}}} \bar{r}(\bar{p}-1) \mathrm{d} \bar{r} \mathrm{~d} \bar{\theta}}{\int_{0}^{1} \int_{\bar{r}_{i}}^{\bar{T}_{T}} \bar{r} \mathrm{~d} \bar{r} \mathrm{~d} \bar{\theta}}
$$

The dimensionless variables $\overline{\Theta_{1}}, \overline{\Theta_{2}}, \ldots \overline{\Theta_{n}}$, $\ldots \overline{\Theta_{n+1}}$, were combined into a vector $v=\left[\overline{\Theta_{1}}, \overline{\Theta_{2}}\right.$, $\left.\ldots \overline{\Theta_{n}}, \ldots \overline{\Theta_{n+1}}\right]$. The maximum LCC was obtained by optimizing the vector $v$. A detailed flow diagram of the processes for the GA-SQP hybrid method is shown in Fig. 2.

The details of the GA and GA-SQP hybrid methods are as follows: the initial population is formulated randomly. One individual chromosome is one vector $v$, which describes the geometry of a single groove

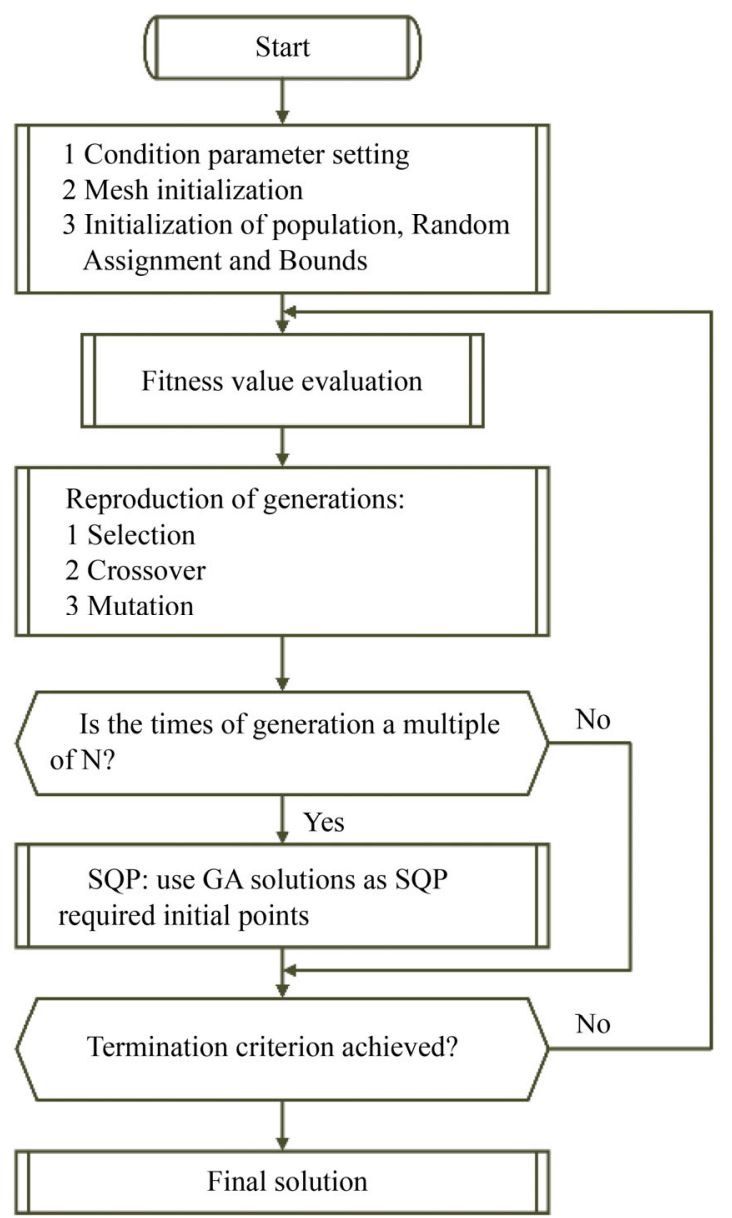

Fig. 2 Detailed flow diagram of the processes for the GA-SQP hybrid method. 
texture. GA optimization results provide initial values for SQP. Local optimization is committed every $N$ evolutionary generations. The GA and SQP are shifted until the calculation convergence occurs. When the optimal value maintains for a certain number of generations (e.g., 20 in this study), we define it as computation convergence. The parameters for the GA-SQP hybrid method, such as the local optimum interval $N$, the population, and the crossover fraction are listed in Table 2.

\subsection{Optimized results}

Taking the rotational speed $\omega=400 \mathrm{rpm}$ as an example for which the geometrical parameters and optimization parameters are listed in Tables 1 and 2, the evolutionary curves are presented in Fig. 3. It can be found that the optimal value of each generation increases continuously at first and then tends to be stable with increasing evolutionary generations. The average value also increases and then gradually approaches the optimal value.

Table 2 Parameter settings for GA-SQP in the simulations.

\begin{tabular}{cc}
\hline Parameters & Settings \\
\hline Individual & $v$ \\
Population & 100 individuals \\
Fitness & Dimensionless LCC solved \\
& with Eq. (6) \\
Crossover fraction & 0.60 \\
Migration fraction & 0.20 \\
$N$, Local optimization interval & 10 \\
\hline
\end{tabular}

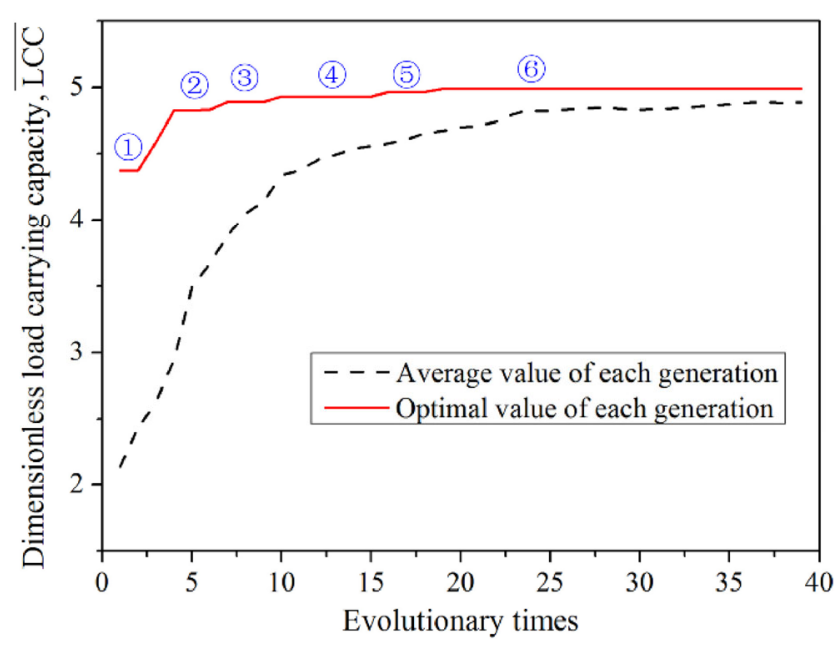

Fig. 3 Evolutionary curves.
The profile of the optimal groove texture changes with increasing evolutionary generations; its evolution is shown in Fig. 4. As the evolutionary generations increase, the profile of the optimal groove texture gradually approaches the chevron.

According to the Stribeck curve, the sliding speed is a key factor affecting the lubrication conditions. In this section, the effects of the rotating speed $\omega$ on the optimized groove texture are described. The numerical optimizations with different rotating speeds of the upper surface ( $\omega=200 \mathrm{rpm}, 400 \mathrm{rpm}, 800 \mathrm{rpm}, 1600 \mathrm{rpm})$ were conducted; the results are shown in Fig. 5.

From the optimization results, it can be seen that the optimized shapes at different rotating speeds are all chevrons. These chevrons are highly similar, and
(1)

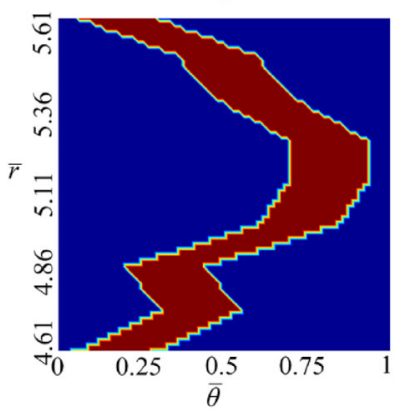

(3)

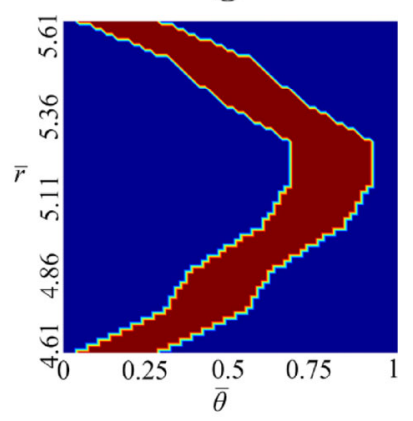

(5)



(2)

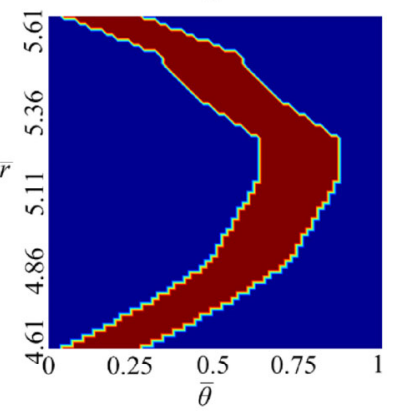

(4)

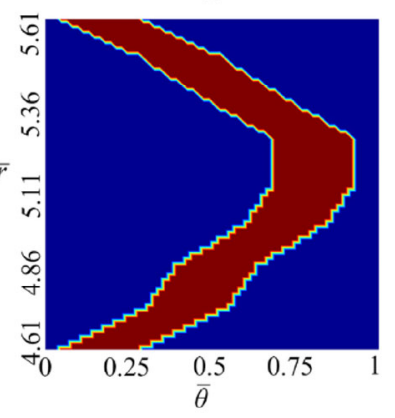

(6)



Fig. 4 Optimal shape evolution process. 
(a) Optimized groove texture



(b) Optimized groove texture

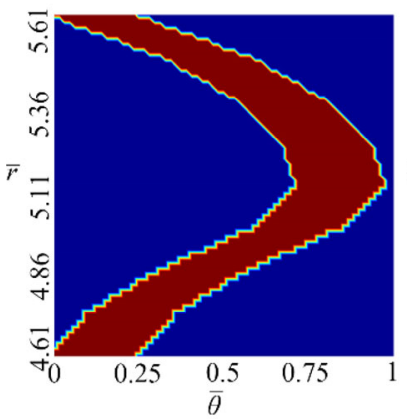

(c) Optimized groove texture

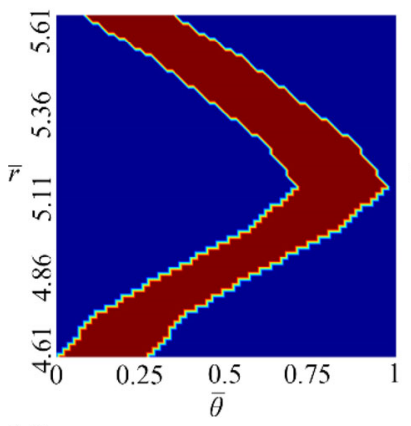

(d) Optimized groove texture

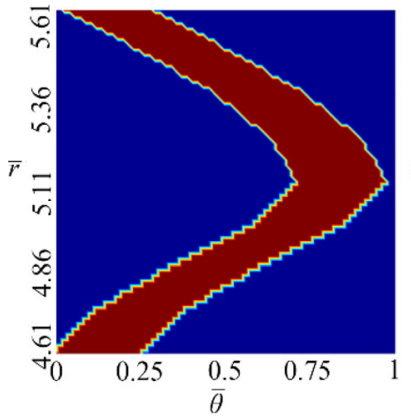

Dimensionless pressure distribution

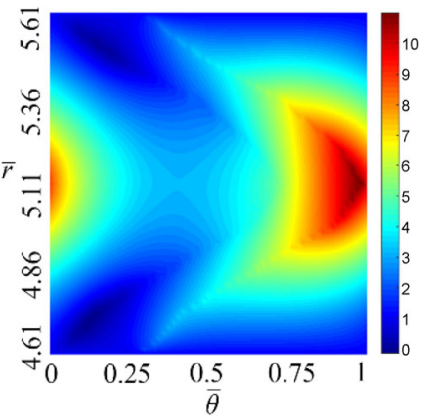

Dimensionless pressure distribution

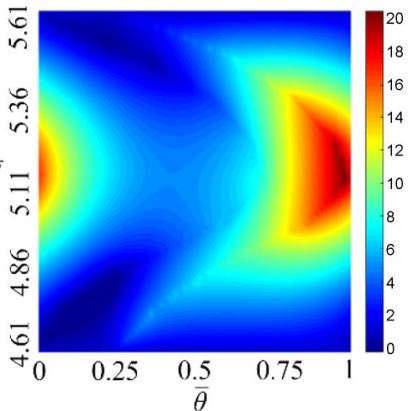

Dimensionless pressure distribution



Dimensionless pressure distribution

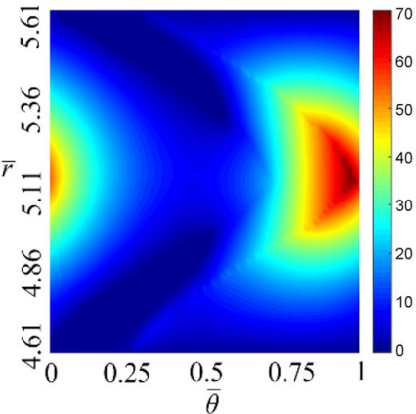

Fig. 5 Optimized results: (a) $\omega=200 \mathrm{rpm}$; (b) $\omega=400 \mathrm{rpm}$; (c) $\omega=800 \mathrm{rpm}$; (d) $\omega=1600 \mathrm{rpm}$.

all the corners orient to the sliding direction. High pressure forms at the corners of the chevrons because of the hydrodynamic effect, and low pressure forms at both ends of the chevrons. The pressure distribution

enhances the LCC of the oil film greatly. The superiority of this profile was validated by experimentation, as described in the next section.

\section{Experiment}

The numerical optimization of surface textures is normally based on a pre-determined oil film thickness. In practical applications, i.e., for thrust bearings and mechanical seals, the oil film thickness is usually unknown, and the load is given. This section describes the friction tests conducted on a standard ring-on-ring friction tester under a pre-set load in order to verify the superiority of the chevrons obtained by the numerical optimization discussed in Section 2.

\subsection{Test rig and lubricant}

In this study, all friction tests were conducted on a standard ring-on-ring friction tester (Plint TE92, Phoenix Tribology, UK), as shown in Fig. 6(a). The material of the rings is $\mathrm{SiC}$, and the surfaces are hard and tough. The untextured upper ring is driven by a rotating shaft with a self-alignment joint, and rotated at a specific speed, $\omega$. The groove-textured bottom ring was fixed and loaded vertically as shown in Fig. 6(b). The frictional torque was measured by a torque sensor, and the surface temperature was obtained with a thermocouple sensor. The geometric parameters of the rings and the testing conditions are listed in Table 3.

During the experiments, both test rings were submerged in the lubricant. The lubricant was PAO 6, and an oil film formed at the interfaces of the rings because of the hydrodynamic effect. The viscosity of the base oil is significantly affected by the temperature. The viscosity-temperature curve was measured with a standard rheometer (Aaton Paar Physica MCR301). The result is shown in Fig. 7 .

\subsection{Specimen preparation and texturing}

Three textured $\mathrm{SiC}$ rings were fabricated and used in these experiments. Three profiles of groove textures, i.e., straight grooves (straight-g for short), large-angle chevrons (large-c for short) and small-angle chevrons (small-c for short), are presented in Fig. 8. Among the profiles, the small-angle chevrons are the result of the 




Fig. 6 Diagrams of the test rig: (a) photograph of the friction tester and (b) schematic of the test rig.

Table 3 Geometric parameters of the rings and testing conditions.

\begin{tabular}{cc}
\hline Item & Value \\
\hline Outside diameter of bottom ring, $D_{\mathrm{o}}(\mathrm{mm})$ & 54 \\
Inside diameter of bottom ring, $D_{\mathrm{i}}(\mathrm{mm})$ & 38 \\
Outside diameter of upper ring, $d_{\mathrm{o}}(\mathrm{mm})$ & 50.5 \\
Inside diameter of upper ring, $d_{\mathrm{i}}(\mathrm{mm})$ & 41.5 \\
Load, $F_{\mathrm{N}}(\mathrm{N})$ & 300 \\
Rotation speed, $\omega(\mathrm{rpm})$ & $200-1000$ \\
Lubricant & PAO 6 \\
\hline
\end{tabular}

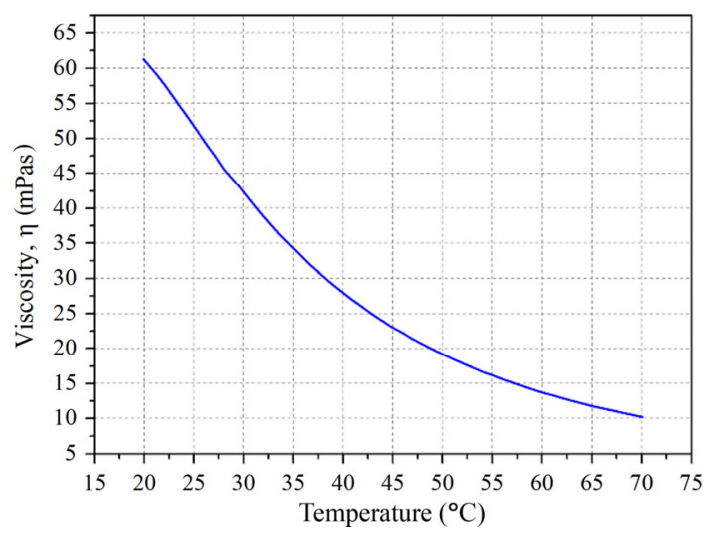

Fig. 7 Viscosity-temperature curve of PAO 6. optimization described in Section 2. These groove textures are periodically distributed along the circumference of the rings. The corners of the chevron grooves are located on a circle with a radius of $r_{\mathrm{c}}$. The profiles of the groove textures were simplified on the basis of optimization results for ease of processing.

On the basis of the periodic boundary conditions, a single groove was extracted as a unit cell as shown in Fig. 9. The chevron grooves are defined with several parameters, which involve the unit cell angle $\theta_{0}$ and the groove texture angles $\theta_{1}, \theta_{2}$. These geometric parameters are listed in Table 4 . The designed AR of the bottom rings in this study remained constant at 0.25 , although this value may vary slightly for different specimens after laser surface texturing.

The topographies of the samples were assessed with a white-light interferometer (Nexview, Zygo, USA). The surfaces of the rings were finely polished. A picosecond laser processing system (PX100-2-GH, Edgewave) was adopted to fabricate the groove textures of the bottom testing rings. The topographies of the groove textures are shown in Fig. 10. There was no
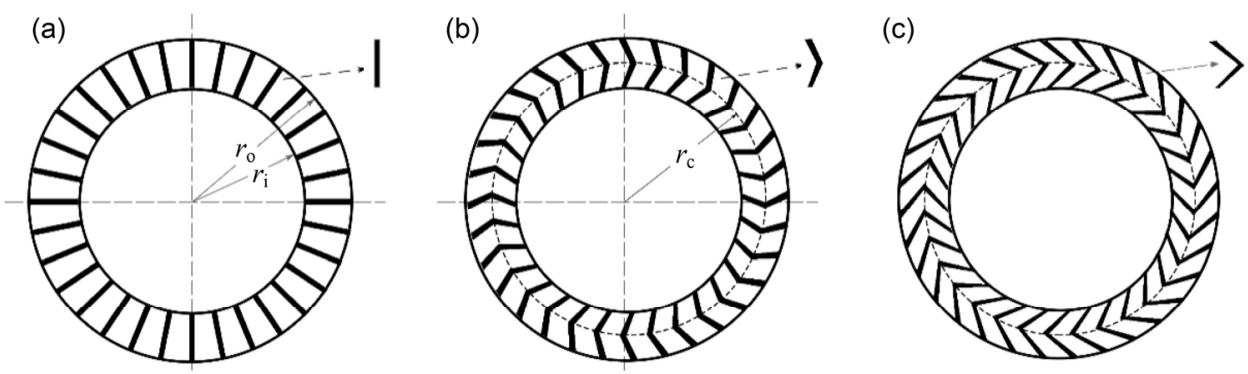

Fig. 8 Profiles of groove-textured surfaces: (a) textured surface with straight grooves; (b) textured surface with large-angle chevrons; and (c) textured surface with small-angle chevrons. 
(a)

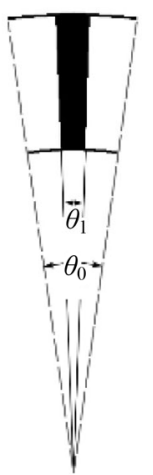

(b)

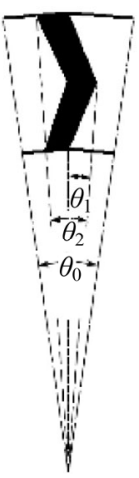

(c)

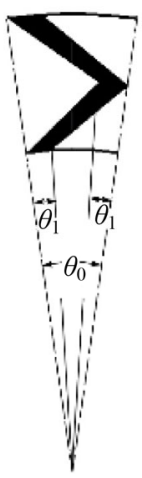

Fig. 9 Profiles of unit cells: (a) straight groove; (b) large-angle chevron; and (c) small-angle chevron (optimized profile).

Table 4 Geometric parameters of the groove textures.

\begin{tabular}{cc}
\hline Parameter & Value \\
\hline Number of groove textures, $n_{\mathrm{t}}$ & 32 \\
Corner radius, $r_{\mathrm{c}}(\mathrm{mm})$ & 23 \\
Unit cell angle, $\theta_{0}\left(^{\circ}\right)$ & 11.25 \\
Groove texture angle, $\theta_{1}\left(^{\circ}\right)$ & 2.81 \\
Groove texture angle, $\theta_{2}\left(^{\circ}\right)$ & 5.63 \\
\hline
\end{tabular}

obvious burr on the edges of the groove textures, so there was no need for secondary polishing. The roughness of the fine polished surface reached $0.014 \mu \mathrm{m}$, as shown in Fig. 11(a). After laser texturing, the roughness of the groove textures' bottom increased to $0.488 \mu \mathrm{m}$, as shown in Fig. 11(b). The depth of all the groove textures was approximately $4.5 \mu \mathrm{m}$.

\subsection{Experimental procedure}

Before the experiments, the specimens were ultrasonically cleaned in an acetone bath, an anhydrous ethanol bath, and a deionized water bath for 5 min each. Then, after the assembly of the specimens was completed, the test was run in a sequence at rotating speeds of $200 \mathrm{rpm}, 300 \mathrm{rpm}, 400 \mathrm{rpm}, 600 \mathrm{rpm}, 800 \mathrm{rpm}$, and $1000 \mathrm{rpm}$ for $5 \mathrm{~min}$ under a constant load of $300 \mathrm{~N}$. When a test sequence was completed, the lubricant and the specimens were all replaced with new objects. In addition to the rings with the three types of groove textures, an untextured ring was also tested. (a1)



(a2)

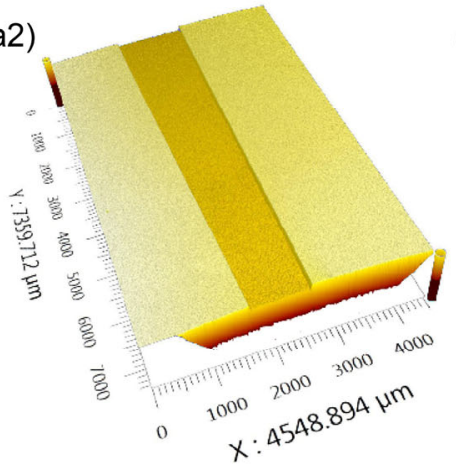

(b1)

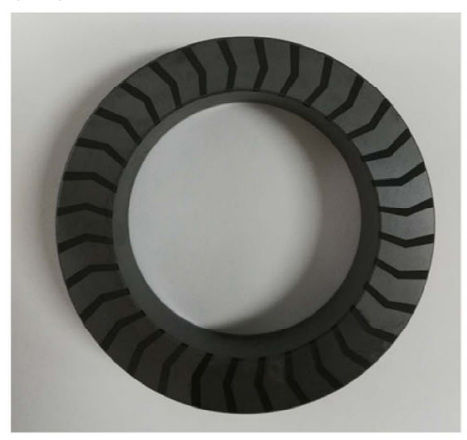

(b2)

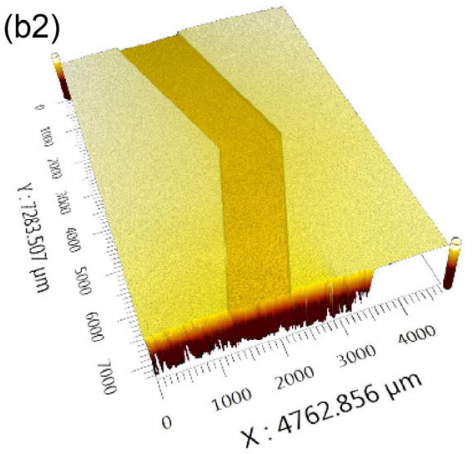

(c1)
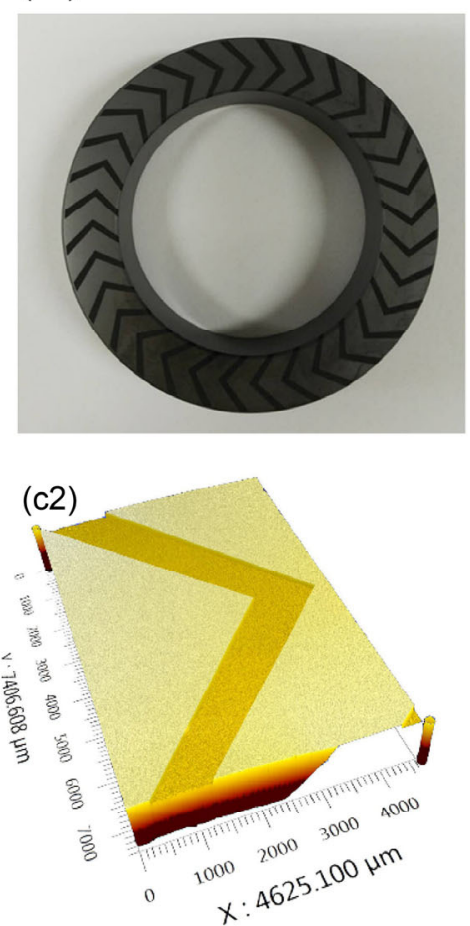

Fig. 10 Topographies of groove textures: (a1) photograph of the ring with straight grooves; (b1) photograph of the ring with large-angle chevrons; and (c1) photograph of the ring with small-angle chevrons; (a2) measured surface topography of a single straight groove; (b2) measured surface topography of a single large-angle chevron; and (c2) measured surface topography of a single small-angle chevron. 

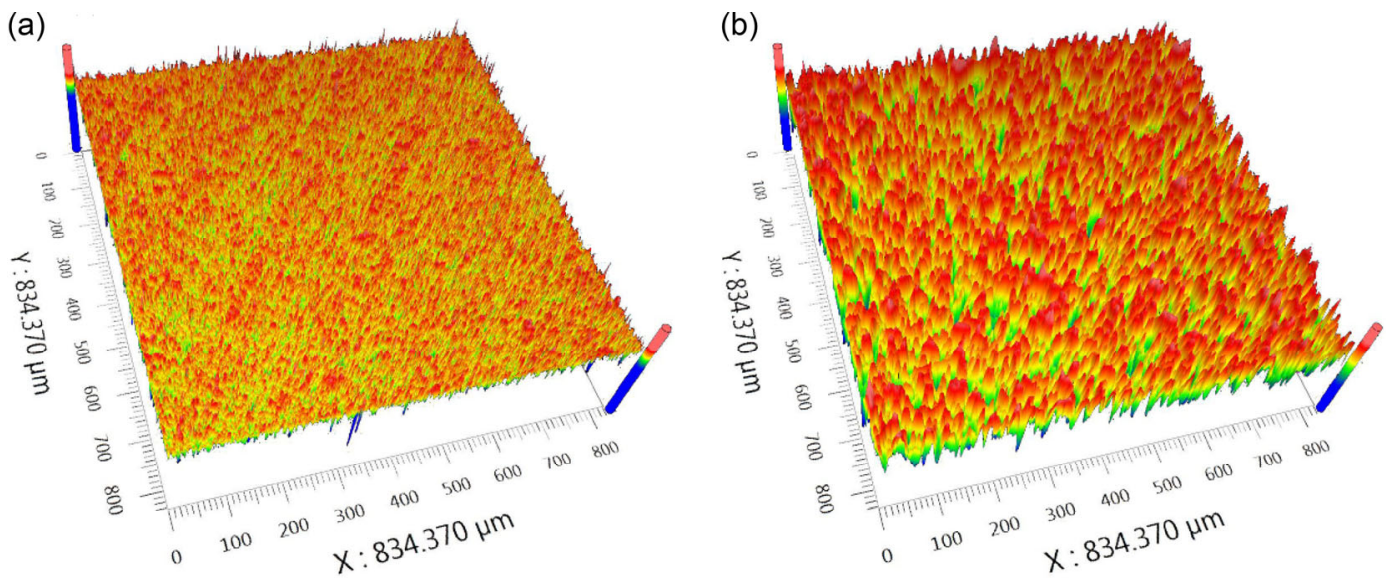

Fig. 11 Measured surface topographies: (a) fine-polished surface and (b) laser-textured surface.

\section{Results, analysis, and discussion}

The COFs of the different rings are shown in Fig. 12. The COFs of the different groove textures are obviously different, as shown in the figure. The COF of the untextured surface is the highest. As the rotating speed increases, the COF decreases. It can be inferred that the lubrication state of this friction pair is boundary lubrication. Compared with the untextured surface, the straight groove textures obviously reduce the COF. As the rotating speed increases, the COF decreases slightly. The LCC of the lubricant is not great enough to separate the two surfaces. The full film does not form and the lubrication state of this friction pair is mixed lubrication. Both of the two types of chevrons significantly reduce the $\mathrm{COF}$, and the friction reduction behavior of the small-angle chevrons is superior over the large-angle chevrons. As the rotating speed increases, the COF increases slightly, and then the lubrication state of these friction pairs is hydrodynamic lubrication. Moreover, the small-angle chevrons, which are also the optimized result, achieve a stable ultra-low COF $(\mathrm{COF}<0.01)$ at the rotating speed of $200 \mathrm{rpm}$.

The temperature rise of the specimen surfaces is generated by the heat caused by friction. Thus, the groove textures reduce the energy loss efficiently while reducing the COF. The temperature profiles of different rings are presented in Fig. 13. After running for $30 \mathrm{~min}$, the temperature rise of the untextured surface was $30^{\circ} \mathrm{C}$, whereas that of the surface with small-angle chevrons was only $5{ }^{\circ} \mathrm{C}$. These results indicate that the optimized groove textures can noticeably reduce the temperature increase.

The oil film thickness was investigated numerically. Considering the fact that the roughness of the untextured surface was $0.014 \mu \mathrm{m}$, it was assumed that the film thickness should be greater than $0.05 \mu \mathrm{m}$ to achieve full film lubrication. Taking into account the


Fig. 12 COFs of different test rings: (a) COF-time curves and (b) COF-rotating speed curves. 

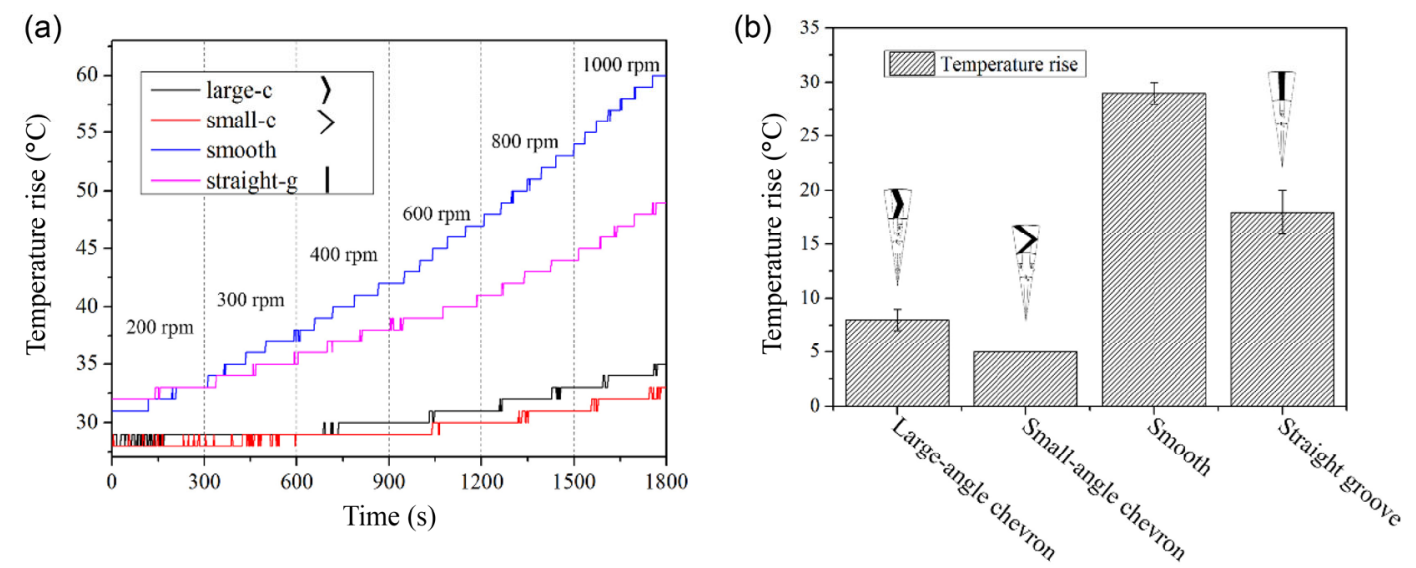

Fig. 13 Surface temperature rise of the rings: (a) temperature-time curves and (b) temperature rise of different test rings.

viscosity-temperature characteristics of the lubricant, the viscosity in Eq. (4) was corrected according to the viscosity-temperature curve shown in Fig. 7. The corrected bearing number $\Lambda$ can be expressed as

$$
\Lambda=\frac{6 \eta(T) \omega}{p_{0}}\left(\frac{r_{o}-r_{i}}{h_{0}}\right)^{2}
$$

For the straight groove textures, the LCC of the oil film was much less than the load when the thickness $h_{0}$ was $0.05 \mu \mathrm{m}$. The pressure distribution $\left(h_{0}=0.05 \mu \mathrm{m}\right.$ and $\omega=200 \mathrm{rpm}$ ) is presented in Fig. 14(a). Significant cavitation occurred in the straight-groove texture. It is the main mechanism of the improvement in the LCC caused by straight groove textures that cavitation suppresses the generation of negative pressure in the oil film. Because the LCC of the oil film is not large enough to support the load, the main mechanism of the reduction in COF caused by straight groove textures is that the textures reduce the local contact area, rather than a result of the hydrodynamic effect.
For the chevron-type groove textures, the LCC of the oil film is much greater than the load when the thickness $h_{0}$ is $0.05 \mu \mathrm{m}$. As the oil film thickness increases, the LCC decreases. Therefore, the oil film was solved with dichotomy according to the load. For the large-angle chevron and the small-angle chevron, the pressure distributions (load $=300 \mathrm{~N}$ and $\omega=200 \mathrm{rpm}$ ) are presented in Figs. 14(b) and 14(c), respectively. The results show that the cavitation that occurs at the ends of the small-angle chevrons is suppressed. Comparing Figs. 14(b) and 14(c), it shows that the angle of the chevron also affects the pressure distribution of the oil film.

In addition to the support of the load, some friction pairs also act to seal, where the COF is not the only criterion for evaluating the lubrication characteristics. When the oil film thickness is small, the shear rate of the lubricant is large, and sometimes the solid surfaces even contact directly. However, when the oil film thickness is large, the leakage may increase. The oil film thickness for the chevrons was calculated as shown in Fig. 15(a). (a) Dimensionless pressure distribution



(b) Dimensionless pressure distribution

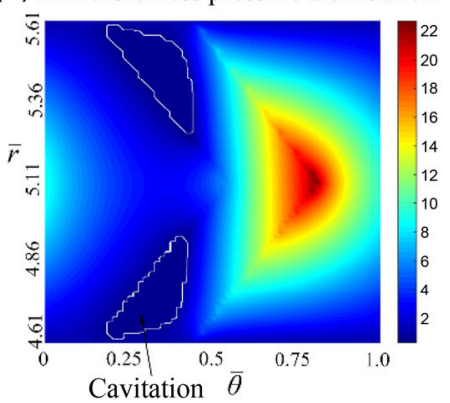

(c) Dimensionless pressure distribution

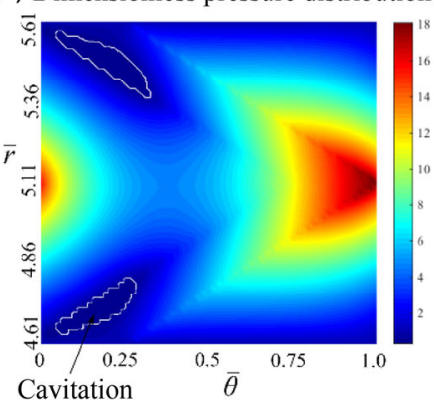

Fig. 14 Dimensionless pressure distribution of computing unit cells: (a) straight-groove texture $\left(h_{0}=0.05 \mu \mathrm{m}, \omega=200 \mathrm{rpm}\right)$; (b) large-angle chevron (load $=300 \mathrm{~N}, \omega=200 \mathrm{rpm})$; and (c) small-angle chevron (load $=300 \mathrm{~N}, \omega=200 \mathrm{rpm})$. 
(a)

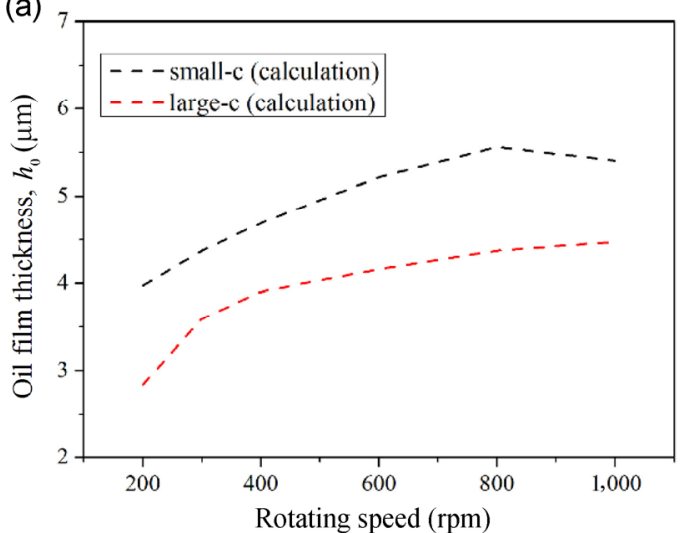

(b)

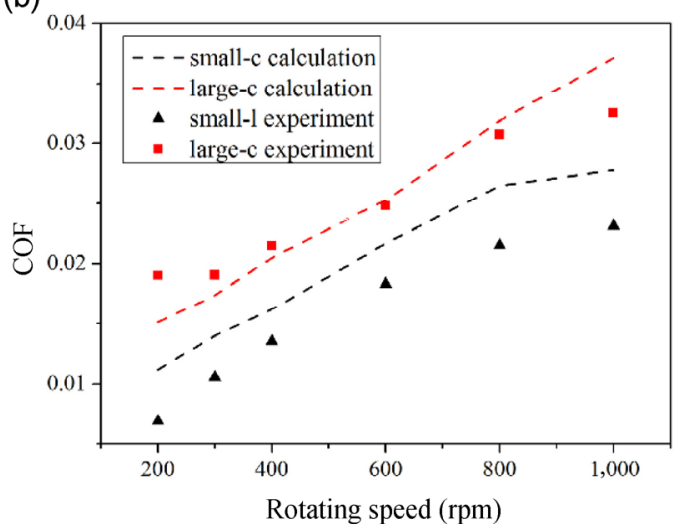

Fig. 15 Calculation and experimental results for chevrons: (a) oil film thickness and (b) coefficient of friction.

The oil film thickness of the small-angle chevrons is larger than that of the large-angle chevrons, which reveals why the COF can be reduced efficiently by the optimized groove textures.

$$
\begin{gathered}
\tau=\frac{1}{2 r} \frac{\partial p}{\partial \theta} h+\omega r \psi \frac{\eta(T)}{h}, \bar{\tau}=\frac{\tau}{p_{0}}, \\
\overline{F_{f}}=\frac{\int_{0}^{1} \int_{\bar{r}_{i}}^{\bar{r}_{o}} \bar{r} \overline{\mathrm{r}} \bar{r} \mathrm{~d} \bar{\theta}}{\int_{0}^{1} \int_{\bar{r}_{i}}^{\overline{r_{o}}} \bar{r} \mathrm{~d} \bar{r} \mathrm{~d} \bar{\theta}}, \\
\operatorname{COF}=\frac{\overline{F_{f}}}{\overline{L C C}}
\end{gathered}
$$

In order to validate the calculated oil film thickness, the COF can be obtained using Eq. (8). The calculated COFs agree well with the experimental results, as shown in Fig. 15(b).

\section{Conclusions}

On the basis of the calculations and experiments, conclusions were drawn as follows:

1) The GA-SQP hybrid method can be applied to optimize the groove texture's profile to improve the hydrodynamic lubrication characteristics. Under certain conditions, the optimized groove textures can achieve a stable ultra-low $\mathrm{COF}(\mathrm{COF}<0.01)$.

2) Small-angle chevrons show significant superiority over other shapes.

3) The main LCC enhancement mechanisms of straight-groove texture and chevrons are different.
For straight-groove textures, the textures reduce the local contact area. For the chevrons, the high pressure generated by the hydrodynamic effect at the corners rather than cavitation is the main reason for the improvement in the LCC of the oil film.

This finding can be widely applied to situations that must consider both friction and leakage, such as mechanical seals and port plate pairs in axial piston pumps.

Open Access: The articles published in this journal are distributed under the terms of the Creative Commons Attribution 4.0 International License (http://creativecommons.org/licenses/by/4.0/), which permits unrestricted use, distribution, and reproduction in any medium, provided you give appropriate credit to the original author(s) and the source, provide a link to the Creative Commons license, and indicate if changes were made.

\section{References}

[1] Hamilton D B, Walowit J A, Allen C M. A theory of lubrication by microirregularities. J Basic Eng 88(1): 177-185 (1966)

[2] Brizmer V, Kligerman Y, Etsion I. A laser surface textured parallel thrust bearing. Tribol Trans 46(3): 397-403 (2003)

[3] Wang X L, Kato K, Adachi K, Aizawa K. Loads carrying capacity map for the surface texture design of $\mathrm{SiC}$ thrust bearing sliding in water. Tribol Int 36(3): 189-197 (2003)

[4] Wakuda M, Yamauchi Y, Kanzaki S, Yasuda Y. Effect of surface texturing on friction reduction between ceramic and steel materials under lubricated sliding contact. Wear 254(3-4): 356-363 (2003) 
[5] Scaraggi M, Mezzapesa F P, Carbone G, Ancona A, Sorgente $\mathrm{D}$, Lugarà $\mathrm{P} \mathrm{M}$. Minimize friction of lubricated laser-microtextured-surfaces by tuning microholes depth. Tribol Int 75: 123-127 (2014)

[6] Tang W, Zhou Y K, Zhu H, Yang H F. The effect of surface texturing on reducing the friction and wear of steel under lubricated sliding contact. Appl Surf Sci 273: 199-204 (2013)

[7] Vlădescu S C, Olver A V, Pegg I G, Reddyhoff T. Combined friction and wear reduction in a reciprocating contact through laser surface texturing. Wear 358-359: 51-61 (2016)

[8] Wang W, He Y Y, Zhao J, Li Y, Luo J B. Numerical optimization of the groove texture bottom profile for thrust bearings. Tribol Int 109: 69-77 (2017)

[9] Etsion I. State of the art in laser surface texturing. J Tribol 127(1): 248-253 (2005)

[10] Siripuram R B, Assistant R, Stephens L S. Effect of deterministic asperity geometry on hydrodynamic lubrication. $J$ Tribol 126(3): 527-534 (2004)

[11] Yu H W, Wang X L, Zhou F. Geometric shape effects of surface texture on the generation of hydrodynamic pressure between conformal contacting surfaces. Tribol Lett 37(2): 123-130 (2010)

[12] Ren N. Advanced modeling of mixed lubrication and its mechanical and biomedical applications. Ph.D Thesis. Evanston, Illinois (USA): Northwestern University, 2009

[13] Yao S M, Ma H W, Wang L Q. Aerostatic \& aerodynamic performance of a herringbone thrust bearing: Analysis and comparisons to static load experiments. Tribol Trans 54(3):

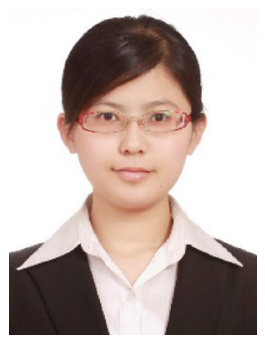

Wei WANG. She received her bachelor degree in mechanical engineering in 2012 from Tsinghua University, Beijng, China. After then, she was a Ph.D student in

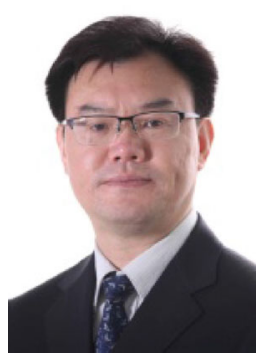

Yongyong HE. He received his M.S and Ph.D degrees in mechanical engineering from Southeast University, Nanjing, China, in 1994 and 1997, respectively. He joined the State
370-83 (2011)

[14] Fesanghary M, Khonsari M M. On the optimum groove shapes for load-carrying capacity enhancement in parallel flat surface bearings: Theory and experiment. Tribol Int 67: 254-262 (2013)

[15] Shen C, Khonsari M M. Numerical optimization of texture shape for parallel surfaces under unidirectional and bidirectional sliding. Tribol Int 82: 1-11 (2015)

[16] Shen C, Khonsari M M. Texture Shape optimization for seal-like parallel surfaces: Theory and experiment. Tribol Trans 59(4): 698-706 (2016)

[17] Shi X, Ni T. Effects of groove textures on fully lubricated sliding with cavitation. Tribol Int 44(12): 2022-2028 (2011)

[18] Zhang J Y, Meng Y G. Direct observation of cavitation phenomenon and hydrodynamic lubrication analysis of textured surfaces. Tribol Lett 46(2): 147-158 (2016)

[19] Elrod H G, Adams M. A computer program for cavitation. In Proceedings of the First Leeds-Lyon Symposium on Cavitation and Related Phenomena in Lubrication, New York, USA, 1974: 37-41.

[20] Fesanghary M, Khonsari M M. On self-adaptive surface grooves. Tribol Trans 53(6): 871-880 (2010)

[21] Alt W. Semi-local convergence of the lagrange-newton method with application to optimal control. In Recent Developments in Optimization. Durier R, Michelot C, Eds. Berlin Heidelberg: Springer, 1995: 1-16

[22] Wilson R B. A simplicial algorithm for concave programming. Ph.D Thesis. Boston (USA): Harvard University, Graduate School of Business Administration, 1963

the State Key Laboratory of Tribology at the same university. She has recently obtained her Ph.D degree in mechanical engineering at Tsinghua University. Her research interests include surface texture and hydrodynamic lubrication.

Key Laboratory of Tribology at Tsinghua University from 1999. His current position is an associate professor and doctoral supervisor. His research areas cover the lubrication, material surface modification, and Chemical Mechanical Polishing (CMP). 